\title{
Investigation into initiation and propagation of cracks in the coated surfaces of spur gears with submodelling and irreversible cohesive-zone modelling techniques
}

\author{
Jiling Feng ${ }^{1, *}$, Yi Qin ${ }^{2}$, and Hanshan Dong ${ }^{3}$ \\ 1 Division of Mechanical Engineering, School of Engineering, Manchester Metropolitan University, Manchester M1 5GD, UK \\ 2 Department of Design, Manufacturing and Engineering Management, University of Strathclyde, Glasgow G1 1XJ, UK \\ 3 Surface Engineering, University of Birmingham, Birmingham B15 2TT, UK
}

Received 30 September 2015 / Accepted 10 March 2016

\begin{abstract}
Spur gears are one of the commonly used transmission parts in industry, due to its simplicity in structures and low cost in manufacturing. Due to a performance requirement, a spur gear may be coated with a specific coating arrangement. Under working conditions, the coating on the teeth may be damaged due to contact fatigue, in the forms such as micro-pitting and/or delamination. The failure mechanism of the coated surface under the gearing contact loading has been investigated intensively through experiment. A comprehensive computational model, which could be used to investigate the propagation of cracks in the coated surfaces, is still lacking. In the research reported in this paper, several finite element modelling techniques, including that for submodelling and irreversible cohesive zone modelling (CZM), have been developed to investigate the failure mechanisms of the coated surfaces of gears under the gearing contact fatigue loading. These techniques not only allow the localized stresses distribution and deformation in the interested locations in the coating and the substrate to be investigated in detail but also enable visual observation on the development of fatigue damages in the coating.
\end{abstract}

Key words: Coated surface, Spur gear, Fatigue damage, FE modelling, Submodelling, Cohesive-zone modelling

\section{Introduction}

Thin, hard PVD (physical vapour deposition) and CVD (Chemical Vapour Deposition) coatings have been widely used in a variety of industrial applications, due to the enhanced hardness and wear resistance of the contact surface [1]. Thin hard coatings have positive influences on the fatigue and corrosion fatigue behaviours of the mechanical components, which justifies their uses in some critical systems such as automotive systems [2] and aeronautic systems [3]. Prior to the initiation of the research reported in this paper, it was found that there was a need to develop a more reliable, theoretical and numerical model for the analysis of coating surfaces in the gears, although some effort had been made on the analysis of the fatigue damages of the coated gears [4, 5]. To-date, analysis of the coatings in engineering surfaces still largely relies on the uses of empirical formulas or is based on experience. Clearly, a more accurate computational model, allowing for the specific working conditions to be taken into account, is much needed from the point of view of the coating design

*Corresponding author: j.feng@mmu.ac.uk and analysis. Most recently, an innovative modelling method, which combined with the parameterized modelling, submodelling technique and cohesive zone model (CZM), was developed to determine the load-bearing capacity for the coated products $[6,7]$. This comprehensive modelling method allows for many key factors, including geometry, material properties and pre-heated treatment, to be considered in the coating design and analysis. This method and model have been validated by a series of micro-indentation tests and gearing contact tests $[6,8]$. In this study, the innovative modelling method was developed further by implementing irreversible cohesive law with unloading-reloading hysteresis, with which the failure mechanisms of the coated spur gear under the contact fatigue loading has been investigated.

\section{Localizations of the damage on the tooth flank}

Based on a conventional standard procedure for gear design, pinions are more apt to pit than gears [9]. This is because the sliding motion of the pinion tends to pull metal 

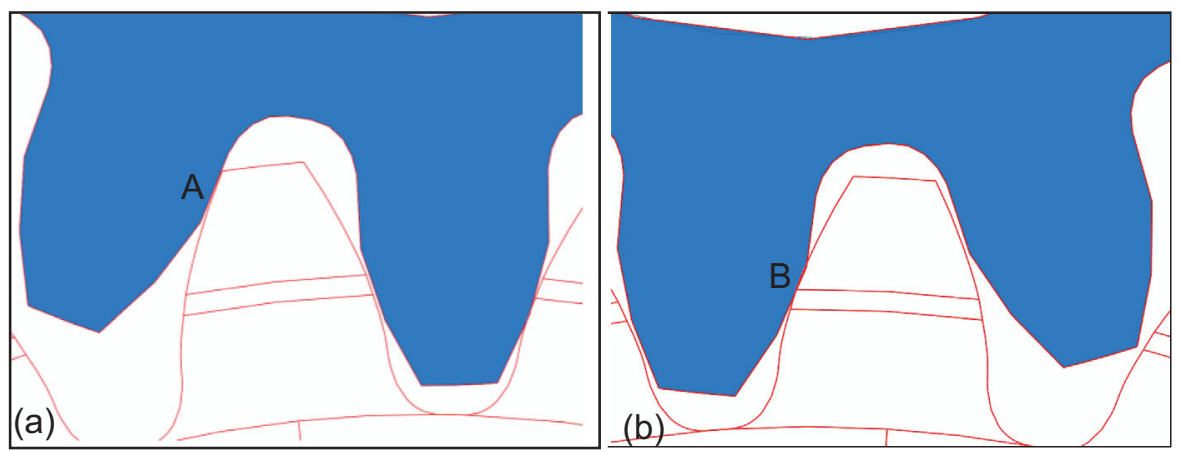

Figure 1. (a) Point A represents the location where the pinion starts to engage with the wheel; (b) Point B represents the location where only one pair of teeth is subjected to the load.

away from the pitch line due to its role as a driver. In addition, the pinion has more cycles of operation than the gear wheel and therefore more apt to fail due to fatigue damage. In this study, investigation of damage behaviour only focuses on the teeth flank of the pinion.

In the teeth flank of pinion, there are two locations where micro-pitting is most likely to occur due to the severe contact loading conditions. These two locations are based on the two extremely points (point A and point B in Figure 1). The point A refers to the location, where the wheel tip touches pinion root and engagement begins. As the pinion has small number of teeth, the radius of curvature of involute profile at the root tends to reduce toward zero, which induces extremely high localized stresses, and hence gives rise to pitting away of the metal. Point B is a typical location, where the load is only supported by one pair of teeth, and hence the highest contact stress is expected to occur. Therefore, Point B is considered as the area where the micro-pitting is easily to occur. In the following sections, the damage performance such as localized contact stresses, plastic deformation and cracks at these locations under the cyclic loading, will be investigated using the developed methodology.

\section{Methodology}

In this paper, the fatigue damage of the coated surface of a pair of spur gears was investigated using the newly developed modelling procedure, which combines several modelling techniques, including the parameterised FE modelling, irreversible CZM and sub-modelling. Parameterised modelling allows for materials and geometrical parameters as well as loading conditions to be described using parameters, enabling many key factors to be incorporated in the analysis as a whole. CZM enables the reproduction of the initiation and propagation of cracks in the coating-layer under complex gear/pinion contact loading conditions. The sub-modelling helps to scale down the geometry of the global model from the macro-scale to the micro/ nano-scale. The sub-modelling is a powerful tool for developing understandings of the performance of coating structures and its failure mechanism in a great detail. The case examined in this study was line contact between a pair of gears, where pinion and wheel have 16 and 24 teeth, respectively. The central distance between gears is $91.1 \mathrm{~mm}$, where addendum

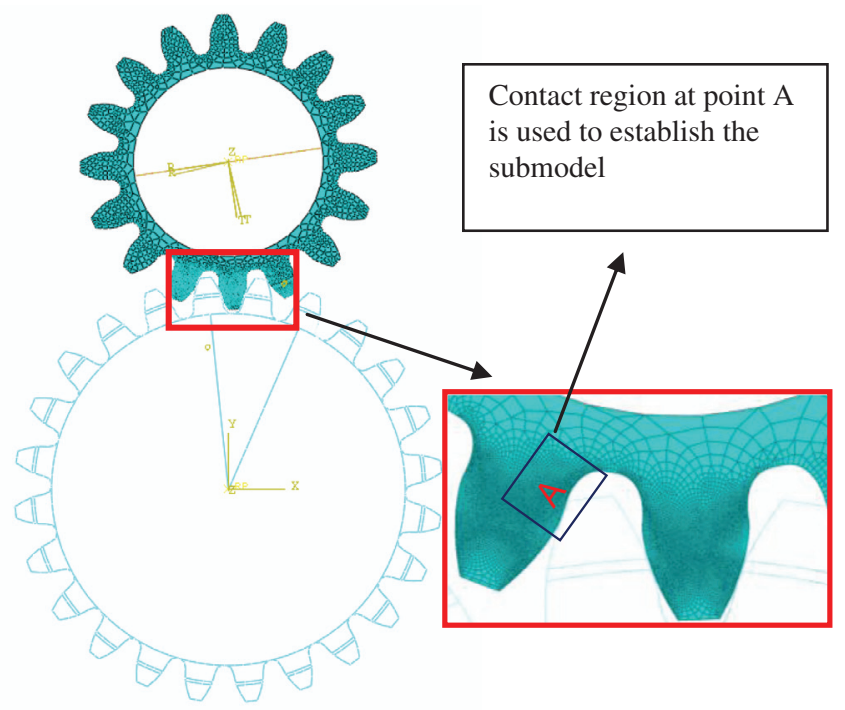

Figure 2. Engagement of the pinion and wheel, where the gear starts to touch root of tooth of pinion (point A) and the engagement starts. The sub-model, representing the contact around the point A, is created, in order to analyze localized contact stresses and crack propagation at this area.

diameter for pinion is $82.405 \mathrm{~mm}$ and for wheel is $118.417 \mathrm{~mm}$. The surface of the gears will be deposited a very thin coating layer with the thickness of 2 micron. Evidently, there is significant difference existed in the geometrical dimensions of gears and thickness of coatings, which causes difficulty in the establishing of a unified model to consider different length scales. In order to address the significant difference of dimensions between the wheel/pinion and coating thickness, a two-step analytical procedure was used: (1) determining the contact conditions (pressure and friction) in the contact area using a global model; and (2) generating a representative sub-model within the contact region so that coating performance can be investigated at the micron-scale level. The techniques described above was shown in Figure 2, where a finite element global model is used to capture the features of geometrical shape and engagement of gears and a sub-model is used to predict the detailed performance of the coated surface. It is scaled down to a micron level where cohesive elements 


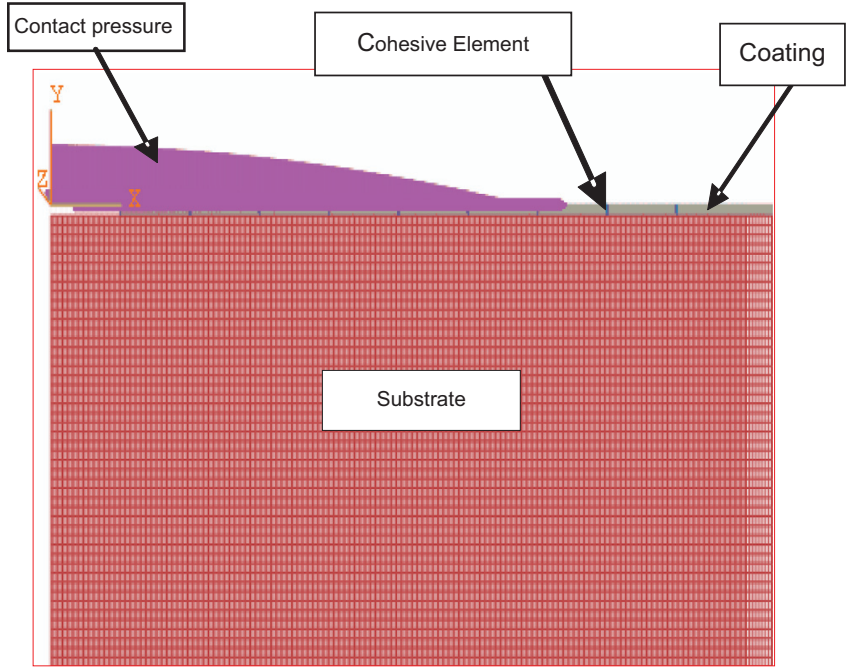

Figure 3. A sub-model, focusing on the contact region at the surface of the pinion in the global model, where each cohesive-zone element was inserted between each pair of the continuum elements within the coating.

were introduced in order to predict the performance of the coating system and its damage micro mechanism.

\section{Cohesive zone model}

The cohesive law, specifying the relationship of the traction and separation across the two virtual adjacent surfaces, provides a phenomenological description for the progressive damage and eventual fracture of the material [10]. Implementation of a cohesive zone model between the continuum elements has been proved to be an efficient technique for the simulation of nucleation and propagation of cracks in the coating surface under monotonic loading [1]. An example of incorporating cohesive elements in a sub-model is shown in Figure 3.

As well known, under the fatigue loading, deterioration of the coating material and cohesive strength are expected to be related largely to the loading history. Accordingly, CZM with unloading-reloading hysteresis would need to be implemented in a model, allowing for the fatigue failure mechanisms under the contact cyclic loading to be investigated.

\subsection{Cohesive-zone model under the monotonically loading}

An irreversible bilinear cohesive-zone modelling approach under the monotonically loading was incorporated with the other modelling techniques including parameterized modelling technique and sub-modelling technique to investigate the crack initiation within the coating layer. The bilinear cohesive-zone law, characterised as the linear function of softening relationship of traction-separation of material degradation, can be written as:

$$
G_{\mathrm{c}}=\frac{1}{2} T_{\max } \delta_{\mathrm{c}}
$$

where: $G_{\mathrm{c}}\left(45 \mathrm{~J} / \mathrm{m}^{2}\right)$ is the critical energy-release rate governing the damage evolution and $T_{\max }$ indicates the maximum traction; and $\delta_{\mathrm{c}}(1 \%)$ is the characteristic cohesivezone length to which the separation reaches when the crack surfaces will be generated. A crack in the coating layer may be initiated when the separation reaches the critical value of, $\delta_{0}$, at which point the traction achieves its maximum value, $T_{\max }$. The parameters of cohesive properties, including the energy-release rate, are crucial to the accuracy of simulation of the cracking within the coating and delaminating of a bonding interface. In this study, the adhesive energy at the atomistic level, was calculated using CASTEP software, which provides an alternative way to determine the value of the cohesive properties. It also provides an approach of linking micro- and nano-mechanical modelling. By means of the cohesive-zone modelling, the critical load in terms of the initiation of the first crack could be predicted. More details on the bilinear cohesive constitutive law, the implementation and applications can be found in author's previous work [6].

\subsection{Cohesive-zone model under cyclic loading}

The fatigue can be considered as one of the major causes that induce the failures in many coated surface-systems. Under a fatigue loading condition, the material deteriorates with the time and the prevailing cohesive strength must be related to the loading history. The cohesive constitutive equation described in equation (1) has been approved an efficient way to simulate the crack within the coating and the delamination of the interface between coating and substrate under the monotonic loading [6]. This equation, however, may results in infinite life under cyclic loading since the dissipation mechanism during the process unloading and re-loading is not taken into account. To develop a model that considers cyclic loading, an irreversible cohesive law with an unloadingreloading hysteresis function, as shown in Figure 4, is employed to simulate the mechanical response of the cohesive zone. This cohesive constitutive law, through introduction of damage variable, $D$, enables the degraded cohesive properties [11]. The modified constitutive equation incorporating the damage variable, $D$, is written as:

$$
\left\{\begin{array}{l}
t_{n} \\
t_{s} \\
t_{t}
\end{array}\right\}=\left[\begin{array}{lll}
K_{n n}(1-D) & & \\
& K_{s s}(1-D) & \\
& & K_{t t}(1-D)
\end{array}\right]\left\{\begin{array}{l}
\varepsilon_{n} \\
\varepsilon_{s} \\
\varepsilon_{t}
\end{array}\right\}
$$

With the condition of cyclic loading, the modified cohesive properties, as described above, are considered in the developed FE model by use of an UMAT subroutine in ABAQUS 11. Each cohesive element possesses four nodes and two integration points. Two types of unloading process, described in equations (1) and (2), respectively, were considered in the subroutine programme. The degraded stiffness of cohesive element during the unloading/reloading process is determined by the current state of displacement, where both of the damage mechanism under monotonic and cyclic loading are considered. 


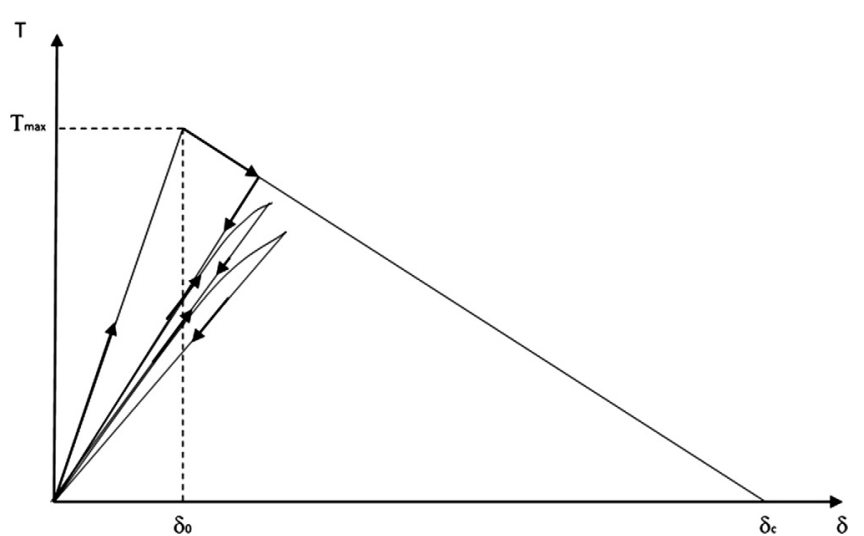

Figure 4. Cohesive traction-separation law under cyclic loading.

Table 1. Material properties of coating and substrate.

\begin{tabular}{ccc}
\hline Materials & $\begin{array}{c}\text { TiN/CrN } \\
\text { coating }\end{array}$ & Substrate \\
\hline Thickness (micron) & 2 & - \\
Hardness (HV) & 1680 & 300 \\
Young's Modulus (GPa) & 240 & 120 \\
Poisson's ratio & 0.3 & 0.3 \\
\hline
\end{tabular}

\section{Results}

\subsection{Configuration of the coating surface of gears}

Crack initiation and propagation analysis had been carried out for a 42CrMo4 spur gear with deposited multilayer TiN/ $\mathrm{CrN}$ coatings. Two different surface systems were investigated in this study and they are defined as the following: (1) Model I: a model PVD TiN/CrN coating on a T-conditioned 42CrMo4, where the substrate was in the simple quenched-and-tempered state; (2) Model II: a model PVD TiN/CrN coating on a nitride $42 \mathrm{CrMo} 4$ substrate.

The spur gears were manufactured in $42 \mathrm{CrMo} 4$ steel and then the gears were super-finished. For the Model I, the steel substrate of spur gear was directly coated with TiN/CrN coating. For the Model II, prior to the coating deposition, the part of steel substrates was subjected to active-screen plasma nitriding, whereas the other part was in the simple quenched-andtempered state. This type of surface system was referred as nitrided $42 \mathrm{CrMo} 4$ substrate. Coating deposition was performed with an industrial cathodic arc evaporation PVD system. The configurations of the two types of gears are summarizes as:

- Model I: PVD TiN/CrN coating on a T-condition 42CrMo4,

- Model II: PVD TiN/CrN coating on a nitride $42 \mathrm{CrMo} 4$ substrate.

In both FE global and the sub-model, the coating and substrate are characterised as homogenous elastic plastic material. The depth-dependent hardness of the hardened layer (after case-hardening) was converted into yield strength, which was
Table 2. Critical loads for two types of surface systems.

\begin{tabular}{cccccc}
\hline Critical load for crack initiation & \multicolumn{2}{c}{ Model I } & & \multicolumn{2}{c}{ Model II } \\
\cline { 2 - 3 } \cline { 5 - 6 } & Point A & Point B & & Point A & Point B \\
\hline Maximum pressure (MPa) & 1438 & 1534 & & 3768 & 3245 \\
Average pressure (MPa) & 1321 & 1279 & & 3331 & 2918 \\
Normal force (N) & 1644 & 5014 & & 4883 & 10,588 \\
Torque (Nm) & \multicolumn{2}{c}{170} & & \multicolumn{2}{c}{358} \\
\hline
\end{tabular}

obtained by a least square fitting-technique, and the strength values were defined with a field method in the FE model.

The main material properties for both substrate and coating are given in Table 1.

\subsection{Computational and experimental results}

To determine the critical load at which a crack was initiated, different levels of loading (Toque) were applied on the global model (pinion/gear system). The pressure distribution at the contact region (point $\mathrm{A}$ and point $\mathrm{B}$ ) from the global model was then transferred to the sub-model as a boundary condition. The critical loads predicted through the FE simulation for two types of configurations of the gears are shown in Table 2. An example of crack propagation in the surface of coating as the loading cycles increases is shown in Figure 5.

The results from the FE model in this study were compared with the experimental results obtained from DGMK-FZG (Deutsche Wissenschaftliche Gesellschaft für Erdöl, Erdgas und Kohle, Forschungsstelle für Zahnräder and Getriebebau der TU München) short micro-pitting test. In such tests, different levels of load (Torque) were applied on the pinion/gear system. During the experiment, the critical loads were defined as the loads under which surface cracks nucleate, where the cracks were observed with scanning electron microscopy (SEM) (Figure 6).

\section{Discussion}

As shown in Table 2, average pressure at point A was greater than that at point $\mathrm{B}$ for both types of gears although the normal force acted on this region was much smaller than that in the region around point $\mathrm{B}$. The reason for that was the small radius of curvature of involute profile at the root leading to the high localized stresses and eventually giving rise to early nucleation of a crack in this area. It was found from both experimental and computational results that pre-treatment with plasma nitriding improved the surface properties dramatically, where the critical load associated with initiation of the first crack for the Model II system is nearly twice higher than that for the Model I system. The greater critical load with the $\mathrm{TiN} / \mathrm{CrN}$ on the nitrided $42 \mathrm{CrMo} 4$ substrate is largely due to the improved strength in the hardened case. As a consequence, the load-bearing capacity is increased for such a system.

The critical loads predicted from the simulation results in both conditions are greater than from experimental test, for instance, the normal force predicted in point B with Model I coated teeth is $1644 \mathrm{~N}$, which is about $20 \%$ greater than from 

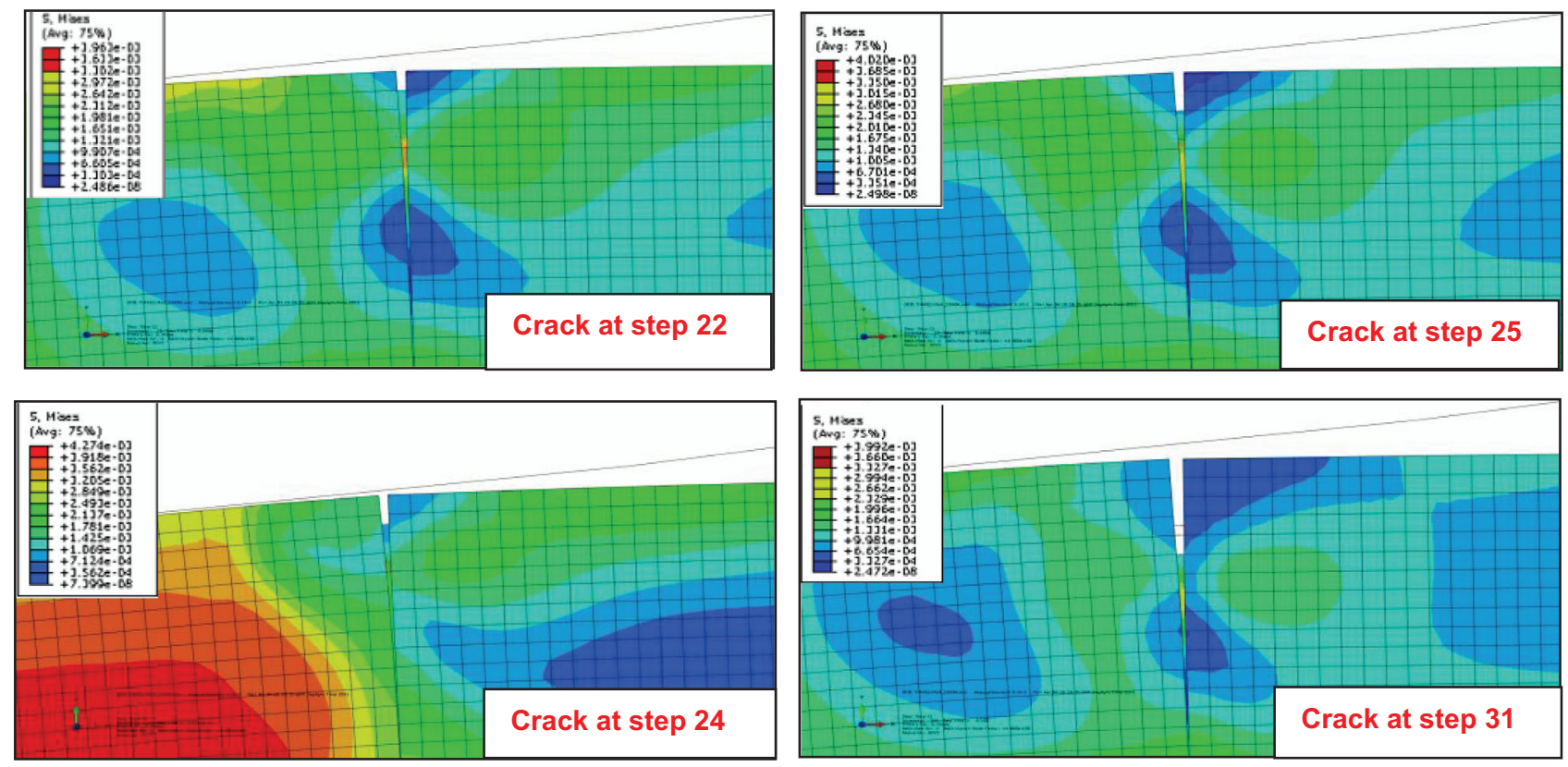

Figure 5. Crack propagation with an increase of the loading cycle.
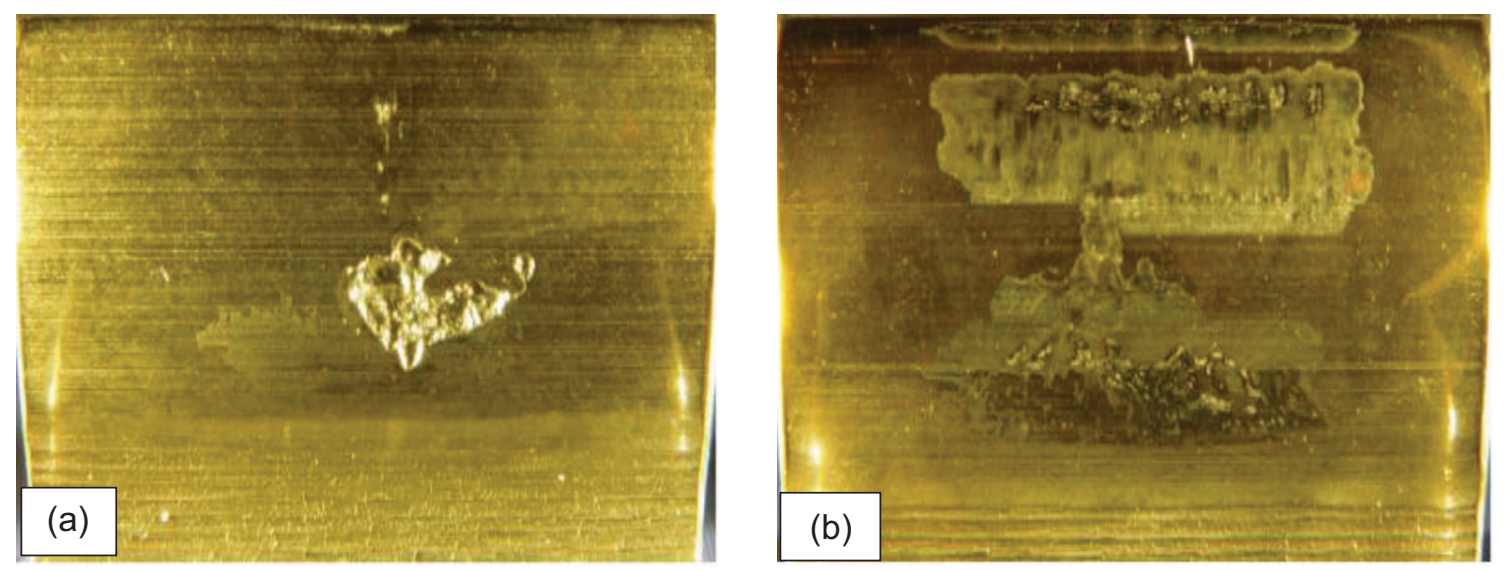

Figure 6. Photographs of the damages in the coated tooth (Model I and Model II). (a) Model I failed at the key loading stage 6 (normal force is $1333 \mathrm{~N}$ in point $\mathrm{A}$ and $3999 \mathrm{~N}$ in point $\mathrm{B}$ ); and (b) Model II failed at the key loading stage 8 (normal force is $2357 \mathrm{~N}$ in point $\mathrm{A}$ and $7072 \mathrm{~N}$ in point $\mathrm{B}$ ).

the experimental test. This discrepancy existed between the experimental and simulation results may be caused by the reason that the lubrication and effect of the roughness of surface were not considered in the FE modelling.

The simulation results also show that the strength of the coated layer degraded with increase of the loading cycles, which led to damages of the coated surface once the crack was nucleated. With an increase of the contact loading cycles, the cracks propagate rapidly to the depth of the coating, which will eventually result in the debonding of the coating from the substrate.

\section{Conclusions}

Several numerical modelling techniques were combined to develop a comprehensive model for the prediction of the failures of the coated surfaces on engineering components, and contact spur-gears were used as a test case in this study. The developed model not only allows localized stress distributions and deformations in the interested locations in coatings and substrate materials to be investigated in detail but also enables visual observation on the development of fatigue damages in the coating, due to the inclusion of a numerical capability of simulating multi-cycle loading in cohesive-zone modelling. The experimental observation on the damages of the coated tooth-surfaces of the gears suggests that similar phenomenon can be predicted with the numerical model developed. Further work is being carried out for improving accuracy of the predicted adhesion energy in coatings and for optimizing the arrangement of cohesive elements in order to further improve accuracy and efficiency of numerical modelling for predicting failures of the coated layers in engineering surface systems. 
Acknowledgements. The research reported in this paper was supported by the EU FP7 project "Multiscale Modelling for Multilayered Surface Systems (M3-2S)", Grant No. CP-FP213600-2 M3-2S. Beneficial discussion with and support from the project partners are acknowledged.

\section{References}

1. S. Baragetti, F. Villa, The Open Materials Science Journal 8 (2014) 87-98.

2. K. Bobzin, N. Bagcivan, N. Goebbels, et al., Surface and Coatings Technology 204 (2009) 1097-1101.

3. A. Feuerstein, A. Kleyman, Surface and Coatings Technology 204 (2009) 1092-1096.

4. S. Baragetti, F. Tordini, Engineering with Computers 27 (2011) 127-137.
5. M. Sraml, J. Falsker, The International Journal of Advanced Manufacturing Technology 31 (2007) 1066-1075.

6. J. Feng, Y. Qin, Q. Zeng, E. Alamandoz, G. Fuente, H. Dong, R. Raghavan, J. Michler, Journal of Multiscale Modelling 1 (2011), 1-22.

7. J. Feng, Y. Qin, Applied Mechanics and Materials 446 (2014) 491.

8. J. Feng, Y. Qin, et al., Steel Research International, Proceeding of Metal Forming (2012), 1059-1062.

9. D.W. Dudley, Handbook of Practical Gear Design, Dudley Engineering Co. McGraw-Hill Book Company, US, 1984.

10. X.P. Xu, A. Needleman, Journal of the Mechanics and Physics of Solids 1397 (1994) 42.

11. A. Abdul-Baqi, P.J.G. Schreurs, M.G.D. Geers, International Journal of Solid and Structure 42 (2005) 927.

Cite this article as: Feng J, Qin Y \& Dong H: Investigation into initiation and propagation of cracks in the coated surfaces of spur gears with submodelling and irreversible cohesive-zone modelling techniques. Manufacturing Rev. 2016, 3, 6. 